NBER WORKING PAPER SERIES

BUBBLES, RATIONAL EXPECTATIONS

AND FINANCIAL MARKETS

Olivier J. Blanchard

Mark W. Watson

Working Paper No. 945

NATIONAL BUREAU OF ECONOMIC RESEARCH

1050 Massachusetts Avenue

Cambridge MA 02138

July 1982

We are indebted to David and Susan Johnson for research assistance, Jeremy Bulow and David Starrett for useful discussion, and to the National Science Foundation, the Sloan Foundation, and the Harvard Graduate Society for financial support. This paper was prepared for the conference on "Crises in the Economic and Financial Structure," New York University, November, 1981. The research reported here is part of the NBER's research program in Economic Fluctuations. Any opinions expressed are those of the authors and not those of the National Bureau of Economic Research. 


\section{Bubbles, Rational Expectations and Financial Markets}

\section{Abstract}

This paper investigates the nature and the presence of bubbles in financial markets.

Are bubbles consistent with rationality? If they are, do they, like Ponzi games, require the presence of new players forever? Do they imply impossible events in finite time, such as negative prices? Do they need to go on forever to be rational? Can they have real effects? These are some of the questions asked in the first three sections. The general conclusion is that bubbles, in many markets, are consistent with rationality, that phenomena such as runaway asset prices and market crashes are consistent with rational bubbles.

In the last two sections, we consider whether the presence of bubbles in a particular market can be detected statistically. The task is much easier if there are data on both prices and returns. In this case, as shown by shiller and singleton, the hypothesis of no bubble implies restrictions on their joint distribution and can be tested. In markets in which returns are difficult to observe, possibly because of a nonpecuniary component, such as gold, the task is more difficult. We consider the use of both "runs tests" and "tail tests" and conclude that they give circumstantial evidence at best.

Olivier J. Blanchard Mark W. Watson Dept. of Economics Harvard University Cambridge, MA 02138 (617) 495-2119 
Introduction

Economists and financial market participants often hold quite different views about the pricing of assets. Economists usually believe that given the assumption of rational behavior and of rational expectations, the price of an asset must simply reflect market fundamentals, that is to say, can only depend on information about current and future returns from this asset. Deviations from this market fundamental value are taken as prima facie evidence of irrationality. Market participants on the other hand, often believe that fundamentals are only part of what determines the prices of assets. Extraneous events may well influence the price, if believed by other participants to do so; "crowd psychology" becomes an important determinant of prices.

It turns out that economists have overstated their case. Rationality of both behavior and of expectations often does not imply that the price of an asset be equal to its fundamental value. In other words, there can be rational deviations of the price from this value, rational bubbles.

The purpose of the paper is twofold. The first is to characterize the conditions under which such a deviation may appear, the shape it may take and the potential implications of such deviations. The second is to investigate how we can discover such deviations empirically. Some of the paper is a review of recent work, but much of it is exploratory in nature and will appear a bit tentative. Although this is no doubt due to shortcomings in the authors' thinking, it may also be due to the nature of these bubbles. They present economists and econometri-. cians with many questions to which they may have little to say.

Some may object to our dealing with rational bubbles only. There is little question that most large historical bubbles have elements of irrationality (Kindleberger [1978] gives a fascinating description of many historical bubbles). Our justification is the standard one: it is hard to analyze rational bubbles: It would be much harder to deal with irrational bubbles. 
Rationality of behavior and of expectations, together with market clearing, imply that assets are voluntarily held and that no agent can, given his private information and the information revealed by prices, increase his expected utility by reallocating his portfolio.

With many more assumptions, this leads to the standard "efficient market" or "no arbitrage" condition.

Let

$$
R_{t}=\frac{p_{t+1}^{\because}-p_{t}+x_{t}}{p_{t}}
$$

then

$$
E\left(R_{t} \mid \Omega_{t}\right)=r \quad \text { or equivalently }
$$

$$
E\left(p_{t+1} \mid \Omega_{t}\right)-p_{t}+x_{t}=r p_{t}
$$

$p_{t}$ is the price of the asset, $x_{t}$ the direct return. We shall refer to $x_{t}$ as the "dividend," although it may take, depending on the asset, pecuniary or non-pecuniary forms. $R_{t}$ is therefore the rate of return on holding the asset, which is the sum of the dividend price ratio and the capital gain. $\Omega_{t}$ is the information set at time $t$, assumed common to all agents. The condition therefore states that the expected rate of return on the asset is equal to the interest rate $r$, assumed constant. Among the assumptions needed to get equation (1) some are inessential and could be relaxed at the cost of increased notational complexity. These are the assumptions of a constant interest rate, no constraints on short sales and risk neutrality. One assumption is, however, of more consequence: it is that, at least after having observed the price, all agents have the same information. As we shall show, bubbles can exist even in this case and these bubbles would remain even if agents have differential information. The question is, however, whether differential information allow's for a larger class of bubbles, and whether some 
aspects of real world bubbles involve differential information. We shall return. to this issue - - with not much to say -- after we define bubbies. (Note also that because of the common information assumption, equation ( 1 ) is stronger than the usual "efficient market" formulation, which is that, for the subset of information common to all agents, $\omega_{t}$, the following relation holds:

$$
E\left(p_{t+1} \mid \omega_{t}\right)-p_{t}+x_{t}=r p_{t} \quad t^{\prime}
$$

Given the assumption of rational expectations and that agents do not forget, so that $\Omega_{t} \subseteq \Omega_{t+1}$, we can solve equation (1) recursively forward, using:

$$
E\left(E\left(\cdot \mid \Omega_{t+i}\right) \mid \Omega_{t}\right)=E\left(\cdot \mid \Omega_{t}\right) \quad . \quad \cdot r i \geq 0
$$

Thus the following $\mathrm{P}_{t}^{*}$ is a solution to equation (1):

$$
p_{t}^{*}=\sum_{i=0}^{\infty} \theta^{i+1} E\left(x_{t+i} \mid \Omega_{t}\right) \quad \theta \equiv(1+r)^{-1}<1 .
$$

$\mathrm{P}^{*}$ is the fresent value of expected dividends and thus can be called the "market fundamental" value of the asset. (The term is standard in financial markets. It was introduced in economics in a similar context by flood and Garber [1980]). $\mathrm{P}^{\star}$ is, however, not the only solution to (1). Any $\mathrm{P}_{\mathrm{t}}$ of the following form is a solution as well:

(3) $p_{t}=\sum_{i=0}^{\infty} \theta^{i+1} E\left(x_{t+i} \mid \Omega_{t}\right)+c_{t}=p_{t}^{*}+c_{t}$, with

$$
E\left(c_{t+1} \mid \Omega_{t}\right)=\theta^{-1} c_{t}
$$

Thus the market price can deviate from its market fundamental value without violating the arbitrage condition. As $\theta^{-1}>1$, this deviation $c_{t}$ must, however be expected to grow over time. (1)

Can this deviation $c_{t}$ embody the popular notion of a "bubble", namely movements in the price, apparently unjustified by information available at the time, 
taking the form of a rapid increase followed by a burst or at least a sharp decline? The following three examples give paths of $c_{t}$ which satisfy equation (3) and seem to fit this notion.

The simplest is that of a deterministic "bubble, " $c_{t}=c_{0} \theta^{-t}$. In this case the higher price is justified by the higher capital gain and the deviations grow exponentially. To be rational, such an increase in the price must continue forever, making such a deterministic bubble implausible. Consider, therefore, the second example:

$$
\begin{aligned}
c_{t} & =(\pi \theta)^{-1} c_{t-1}+\mu_{t} & \text { with probability } \pi \\
& =\mu_{t} & \text { with probability } 1-\pi
\end{aligned}
$$

where $\quad E\left(\mu_{t} \mid \Omega_{t-1}\right)=0$

How will such a bubble look? In each period, the bubble will remain, with probability $\pi$, or crash, with probability $1-\pi$. While the bubble lasts, the actual average return is higher than $r$, so as to compensate for the risk of a crash. The average duration will be of $(1-\pi)^{-1}$. There can be many minor extensions of this example, which also appear to capture certain aspects of bubbles. The probability that the bubble ends may well be a function of how long the bubble has lasted, or of how far the price is from market fundamentals. If $\pi$ increases for some period of time, $c_{t}$ will be growing at a decreas: ing exponential expected rate; if $\pi$ decreases, the higher probability of a crash leads to an acceleration while the bubble lasts.

In these two examples, the büble proceeds independently of the fundamental value. There is no reason for this to be true as the last example shows. Consider a war related stock which pays 1 every period if there is a war, and 0 if there is no war. Suppose a war starts and that in each period there is a probability $\pi$ that the war goes on, a probability $(1-\pi)$ that it 
stops forever. The fundamental value is therefore equal to:

$$
p_{t}^{*}=\sum_{i=0}^{\infty} \theta^{i+1} E\left(x_{t+i} \mid \Omega_{t}\right)=\sum_{i=0}^{\infty} \theta^{i+1} \pi^{i}=\theta(1-\theta \pi)^{-1}
$$

Furthermore, it is constant during the duration of the war. The price may, however, increase above $\mathrm{p}^{\star}$ in anticipation of future increases during the var. For example, the following bubble might arise:

$$
\begin{aligned}
& c_{t}=c_{0} \\
& c_{t+i}=(\theta \pi)^{-1} c_{t+i-1} \text { if there is war at } t+i \text {, } \\
& =0 \text { if there is no war at } t+i \text {. }
\end{aligned}
$$

This will lead to an increase in the price above its fundamental value initially, a further increase during the war, and a crash in both the fundamental value and the bubble when the war ends.

Now that a definition and examples of bubbles have been given, we may return to the simplifying assumptions made to obtain equation (1). What if agents were risk averse? As the last two examples show, bubbles are likely to increase the risk associated with holding the asset. If agents are risk averse, a higher expected return will be required for agents to hold it. Thus, the price will have to be expected to grow even faster than in equation (3). If the probability of a crash increases for example, the price, in the event the crash does not take place, will have to increase faster, not only to compensate for the increased protability of a fall, but also to compensate for the large risk involved in holding the asset.

What if agents do not have the same information? Each agent will then have his onn perception of the fundamental value, given by equation (3), with the agent's 
information set $\Omega_{i t}$ replacing $\Omega_{t}$. As agents may not have the same fundamental value, they will not perceive the same bubble. There will be agent specific bubbles, defined as the difference between the price and the agent's perception of the fundamental value. These bubbles must still satisfy the second part of equation (3), with $\Omega_{i t}$ replacing $\Omega_{t}$ : they must be expected to grow exponentially, at rate $\theta^{-1}$. Could it then be that some agents in the market know that there is a bubble while others do not? A typical speculation scheme of the 1920's (Thomas and Morgan Witts [1979]) was the creation of a high volume of buying by traders having the reputation of being informed, in the hope of creating additional buying by uninformed traders and a subsequent bubble. If such schemes were consistent with rationality of uninformed traders, we might gain insights on how bubbles start. At this stage, however, we do not know the answer (Tirole [1980] makes some progress in this direction). 


\section{Section II Bubbles and transversality conditions}

The previous section has only shown that arbitrage does not by itself prevent bubbles. Could there be, however, other conditions, imposed either institutionally or from market clearing or implied by rationality such that bubbles can in fact be ruled out? This section considers whether such conditions may exist.

As any deviation $c_{t}$ must satisfy condition (3), this implies by successive iteration.

$$
\text { (5) } \begin{aligned}
\lim _{i \rightarrow \infty} E\left(c_{t+i} \mid \Omega_{t}\right) & =+\infty \text { if } c_{t}>0 \\
& =-\infty \text { if } c_{t}<0
\end{aligned}
$$

This is true even for the last two examples. Although the probability that the bubble ends tends to one as the horizon increases, the very large and increasing value of the price if the bubble does not end implies that the expected value of the price increases as the horizon increases.

Condition (5) is clearly impossible to satisfy for any asset redeemable at a given price at a given date. For such assets, the price must equal the par value on that date: the deviation must be zero on that date. Working backwards, the deviation must be zero today. Thus there cannot be bubbles on bonds, except on perpetuities.

Condition (5) also implies that, at least for the model considered here, there cannot be negative bubbles. A negative value of $c_{t}$ today implies that there is a positive probability, possibly very small, that at some time $t+i$, $c_{t+i}$ will be large and negative enough to make the price negative. If the asset can be disposed of at no cost, its price cannot in fact be negative ${ }^{(2)}$; rationality implies that $c_{t}$ cannot be negative today. This argument may, 
however, be pushing rationality a bit too far. For negative values of $c_{t}$, the probability of the price becoming negative may be so small, and the future time so far as to be considered - nearly rationally - irrelevant by market participants.

Apart from institutional boundary conditions, have we exhausted the restrictions imposed by rationality? Bubbles resemble Ponzi games. Ponzi games which grow too fast are inconsistent with rationality. Isn't it the same for bubbles? It may indeed be.

Suppose first that there is a finite number of infinitely lived players market participants. If the price is below the market fundamental, then it will pay to buy the asset and to enjoy its returns - or to rent it out to agents who enjoy it most in each period - forever, i.e. never to sell it again. Thus there cannot be a negative bubble. What if the price is above market fundamentals? With short selling, it will pay to sell the asset short forever and thus again there cannot be a positive bubble. The same result arises, however, even in the absence of short selling. The only reason to hold an asset whose price is above its fundamental value is to resell it at some time and to realize the expected capital gain. But if all agents intend to sell in finite time, nobody will be holding the asset thereafter, and this cannot be an equilibrium. (This point is made more rigorously by Tirole [1980].) Therefore, with rationality and infinitely lived agents, bubbles cannot emerge.

As for Ponzi games, what is needed is the entry of new participants. If a market is composed of successive "generations" of participants, then the above arguments do not hold and bubbles can emerge.

This section ends with another set of intellectual speculations. We have shown where bubbles may exist. 'Can we say where bubbles are more likely to appear? Bubbles are probably more likely in markets where fundamentals are diffi. cult to assess, such as the gold market. If we assume that gold has two uses, one 
industrial use and a precautionary use against major catastrophies, the market fundamentals for gold are the factors affecting future flow industrial demand and flow supply, as well as the determinants of these major catastrophies. These are difficult to assess, as least for the average market participant. He is more likely to base his choice of whether or not to hold the asset on the basis of past actual returns, rather than on the basis of market fundamentals. He may hold gold at a high price because gold has yielded substantial capital gains in the recent past. By the same argument, bubbles are less likely for assets with clearly defined fundamentals such as blue chip stocks or perpetuities. 
Section III Real effects of bubbles

Until now, we have taken the market fundamentals as given, unaffected by the bubble. Bubbles, however, have real effects and do in turn affect market fundamentals, further modifying the behavior of prices.

Bubbles and production of the asset.

If the asset is not reproducible, the bubble will simply lead to rents to the initial holders. Many assets subject to bubbles are, however, partly reproducible. Consider for example housing:

Housing can be thought of as an asset composed of two inputs, 1 and and structures. There is an upward sloping supply curve for land. The supply. of structures is inelastic in the short run, elastic in the long run. In a well functioning market in steady state, the price of houses is equal to the present value of housing services - "rents". In turn, the price and associated return to building structures are such that new housing construction equals the depreciation on the existing stock. (Poterba [1980] formalizes the housing market along those lines, although he does not include land.) Suppose now that a deterministic bubble starts in this market, with agents ready to pay more than market fundamentals. The higher price of housing implies higher returns to housing construction, a larger housing stock in the future, and thus, given an unchanged demand for housing services, lower rents in the future. This implies a decrease in the present discounted value of future rents: the bubble has the effect of immediately decreasing the market fundamental value. What happens over time? The price of housing increases, as the bubble must grow exponentially, leading to a higher and higher housing stock and lower and lower rents. These lower rents are reflected in a lower and lower fundamental value of housing over time, which is simply the symptom of overproduction of housing. The increase in new housing construction may come to an end if land supply becomes entirely 
inelastic, at which point further increases in the price become reflected entirely in land values. If the bubble is not deterministic but stochastic, the story is identical. When the bubble bursts, the price drops to a level lower than the pre-bubble level because of the very large housing stock. Consider finally bubbles in the stock market. Suppose that a firm is initially in equilibrium, with a marginal product of capital equal to the interest rate. In the absence of bubble, the value of a title to a unit of capital, a share, is just equal to the replacement cost and the firm has no incentive to increase its capital stock. Suppose that a bubble starts on its shares, increasing the price, say by $10 \%$ above market fundamentals. Should the firm invest more or should it disregard the stock valuation? One answer is that it should add to the capital stock until the marginal product has been reduced by $10 \%$. When this is done, the market fundamental is decreased by $10 \%$, the share price is again equal to the replacement cost and initial shareholders have made a profit on the new shares issued. The story thereafter is similar to the housing story above, with the fundamental value decreasing while the share value increases. (A more appealing strategy for the firm would be to issue shares and buy shares of other non-bubble firms, therefore avoiding the decrease in marginal product. This strategy is, however, inconsistent with the assumption that the bubble is on titles to capital in the initial firm.) The above answer, however, assumes that the bubble proceeds independently of the actions of the firm. It may well be that the bubble depends on those actions, for. example bursting, if the firm issues "too many" new shares. Again here, there are many stories consistent with rationality and the economist has little to say about which one will prevail. It is therefore not clear how firms should react to bubbles on their stock, and this might explain why maragers of firms seem sometimes to pay little attention to stock market movements. 
General equilibrium effects.

A bubble on the price of any asset will usually affect the prices of other assets, even if they are not subject to bubbles. The increase in the price of the asset which is subject to a bubble leads initially to both an increase in the proportion of the portfolio held in that asset and an increase in total wealth. The first will, if assets are not perfectly substitutable, require an increase in the equilibrium expected return on the asset with a bubble, a decrease in the equilibrium expected return on most other assets. The second effect will, by increasing the demand for goods and possibly for money, lead to an increase in the equilibrium average expected return. The net effect is ambiguous but likely to be a decrease in the price of most of the other assets, together with a further decrease in the fundamental value of the asset experiencing the bubble. A bubble on housing or gold may for example depress the stork market.

Bubbles may therefore have many real effects. This raises a question related to the previous sections. A rational bubble must be expected to grow exponentially. This may imply, when the effects on other markets are taken into account, that some other prices may be expected to grow or decrease exponentially as well. Won't this lead to expected negative prices or some such impossibility, ruling out the existence of a rational bubble in the initial market? The answer is that it depends. If for example, there exists a perfect substitute for a given asset, available in infinite supply at some - possibly. high - price, this prevents a positive bubble on this asset as it puts an upper bound on its price. It is, however, possible to construct general equilibrium models in which bubbles cannot be ruled out. 
Section IV Looking for Bubbles; I

Bubbles can have substantial real effects. It is therefore of some importance to know whether they are a frequent phenomenom or a theoretical possibility. of little empirical relevance.

One strategy is to specify a particular class of bubbles, to assume, for example, that they are deterministic, and to attempt to find whether bubbles of this class exist in a particular market. Although this sometimes may be a sound strategy (such as in the case of the German hyperinflation studied by Flood and Garber [1980]), bubbles can take many forms and specifying a class general enough to include most makes discovery very difficult. A better strategy, and the one we shall explore, is to find evidence of rejection of the "no-bubble" hypothesis, if possible in the direction of the hypothesis of the presence of bubbles. (Rejection of the null hypothesis of "no bubble" may be due to other phenomena than bubbles, such as irrationality.)

We therefore have to deal with two problems. The first is to characterize the restrictions on the behavior of the price, $p$, given the dividend, $x$, under the null hypothesis of no bubbles. The difficulty here is that even if $p$ and $x$ are observable, we usually have no knowledge about the way information on $x$ is revealed to market participants. Information may come infrequently and in lumps; it may come from variables which the econometrician cannot observe, etc. The second problem is to find which of these restrictions are likely to be violated in the presence of bubbles. The difficulty here is the lack of structure on bubbles beyond condition ( 3 ).

If we only have data on $p$, and are unwilling to make any assumptions about the process generating $x$ and the information process, there is no hope of showing the presence or absence of bubbles. Recall that $p$ is a sum of two components, market fundamentals and bubbles. We cannot say something about one of these 
components without knowing something about the other component.

This rather trivial point indicates how difficult it is to prove or disprove the existence of bubbles in a market like gold, where market fundamentals are hard to assess. It also implies that, to make progress we need data on $x$, or assumptions concerning the generation of expectations of $x$, or both.

In the next section we consider tests that can be carried out when only data on $p$ are available. These tests are useful only if one is willing to make strong assumptions about $x$ and the information structure. Their usefulness is therefore severely limited. In this section we consider tests that can be used when data on both $p$ and $x$ are available.

Intuition suggests that bubbles may affect the second moments of $(p, x)$ in two ways. By introducing additional noise, they may increase the variance of $p$. They may also weaken the relation of $p$ to its fundamental determinant $x$ and thus decrease the correlations between $p$ and $x$. We now consider these two intuitions in turn, making them more precise and operational.

\section{The Variance of $p$}

We must distinguish between the unconditional and the conditional variances of $p$, given respectively by:

$$
\begin{aligned}
& v_{u} \equiv E(p-E(p))^{2} \text { and } \\
& v_{c} \equiv E\left(p-E\left(p \mid \Omega_{-1}\right)\right)^{2}
\end{aligned}
$$

Note for future use that although $v_{c}$ involves $E\left(p \mid \Omega_{-1}\right)$; which we do not observe, it is also related, given equation (1), to the variance of the excess return since

$$
v_{c}=E(p(R-r))^{2}
$$

It follows from equation (3) that if a stock is subject to a stochastic bubble, its unconditional variance is indeed infinite. This is not, however, 
necessarily the case for the conditional variance. The excess return, in the presence of a bubble, is given by:

$$
p_{t}-E\left(p_{t} \mid \Omega_{t-1}\right)=n_{t}+\varepsilon_{t}
$$

where

$$
\varepsilon_{t} \equiv c_{t}-E\left(c_{t} \mid \Omega_{t-1}\right)
$$

and

$$
\eta_{t} \equiv \sum_{i=0}^{\infty} \theta^{i+1}\left[E\left(x_{t+i} \mid \Omega_{t}\right)-E\left(x_{t+i} \mid \Omega_{t-1}\right)\right]
$$

so that $\varepsilon_{t}$ is the innovation in the bubble and $\eta_{t}$ is the innovation in the market fundamental value. If $\varepsilon_{t}$ and $\eta_{t}$ are negatively correlated, the excess return could have a lower variance in the presence of a bubble. It is probably safe to assume that for most assets the innovations in the bubble are either uncorrelated or positively correlated with the innovations in $x$, in which case bubbles will increase the variance of the excess return and conditional variance of $\mathrm{p}$.

What are the bounds on the conditional and unconditional variance of $p$ imposed by the hypothesis of no bubble? This question has been analysed by Shiller [1981], who has derived the maximum values for these two variances given the variance of $x$. Given that in practice not only the variance but the autocovariances of $x$ can be estimated, it is easy to tighten his bounds by using this additional information. To derive bounds, we need to make some assumption of stationarity. For notational convenience we will assume that $x_{t}$ has zero mean (since means are unimportant when calculating variances and covariances) and is generated by

$$
x_{t}=\sum_{i=0}^{\infty} \sum_{j=1}^{n} \bar{a}_{i j} \xi_{j t-i}
$$

where $E\left(\xi_{j t}\right)=0, E\left(\xi_{j t}^{2}\right)=i, E\left(\xi_{j t} \xi_{k t-i}\right)=0$, unless $j=k, i=0$ and

$$
v\left(x_{t}\right)=\sum_{i=0}^{\infty} \sum_{j=1}^{n} a_{i j}^{2}<\infty
$$


Although this imposes restrictions on the process generating $x$, it still allows information on $x$ to come in lumps; the variance of the $\xi^{\prime} s$ conditional on the past need not be constant. We place no restrictions on the distribution of the $\xi^{\prime}$ s other than the moment restrictions.

Given these assumptions, and assuming that $\Omega$ contains present and past values of $x_{t}$, two upper bounds are easily derived (see Singleton [1980]). The first is an upper bound on the unconditional variance of $p$ : it is attained if agents know in advance the future values of $x$, i.e. if they have perfect foresight. In this case:

$$
\begin{aligned}
& p_{t}=\sum_{i=0}^{\infty} \theta^{i+1} x_{t+i} \text {, so that } \\
& v_{u}^{\max }=E\left[\left(\sum_{i=0}^{\infty} \theta^{i+1} x_{t+i}\right)^{2}\right] .
\end{aligned}
$$

The second is an upper bound on the conditional variance of $p$. This bound is attained if the information set $\Omega_{t}$ includes only current and past values of $x_{t}$. In this case

$$
p_{t}-E\left(p_{t} \mid \Omega_{t-1}\right)=\sum_{i=0}^{\infty} \theta^{i+1} y_{t+i}
$$

where

$$
y_{t+i} \equiv E\left(x_{t+i} \mid x_{t}, x_{t-1}, \ldots\right)-E\left(x_{t+i} \mid x_{t-1}, x_{t-2} \ldots\right)
$$

so that

$$
v_{c}^{\max }=E\left[\left(\sum_{i=0}^{\infty} \theta^{i+1} y_{t+i}\right)^{2}\right] \text {. }
$$

These upper bounds are likely to be violated when bubbles are present. We can therefore test for bubbles by estimating the actual variances and these upper bounds to see whether they are violated by the data.

Shiller has computed the sample unconditional variance of $p$ and the upper bound given by (7), using annual observations from 1871 to 1979 for real prices, 
and real dividends from the Standard and Poor's index. (The data used are deviations from an exponential trend. See Shiller [1981] for details.) The sample variance, $\hat{v}_{u}$, is 2512 , while the sample estimate of the upper bound variance, $\hat{v}_{u}^{\max }$, is 80 , so that these point estimates clearly violate (7).

To construct a sample estimate of the upper bound of the conditional variance (8), we must first estimate a univariate ARIMA model for the dividend series, $x$. The best fit is achieved by an AR(2) model:

$$
\begin{aligned}
x_{t} & =a_{1} x_{t-1}+a_{2} x_{t-2}+\xi_{t} \\
& =1.07 x_{t-1}-.30 x_{t-2}+\xi_{t}, \hat{\sigma}_{\xi}^{2}=.796
\end{aligned}
$$

The asymptotic variance-covariance matrix of the estimates is

$$
\operatorname{var}\left(\left[\begin{array}{c}
\hat{a}_{1} \\
\hat{a}_{2} \\
\hat{\sigma}_{\xi}^{2}
\end{array}\right]\right)=\left[\begin{array}{ccc}
.0086 & -.0071 & 0 \\
-.0071 & .0086 & 0 \\
0 & 0 & .0074
\end{array}\right]
$$

Diagnostic checks of the model are:

$$
\mathrm{Q}(24)=23.9 ; \mathrm{L}(3)=.850 ; \mathrm{L}(8)=3.96
$$

The $Q$ statistic is a general test for adequacy of the model, and if the model is correctly specified, is distributed $x^{2}(24) . L(3)$ and $L(8)$ are Lagrange Multiplier tests, testing for $A R(3)$ and $A R(8)$ alternatives. If the model $A R(2)$ is correct, they are distributed $x^{2}(1)$ and $x^{2}(6)$. All three tests suggest the $\operatorname{AR}(2)$ specification is adequate.

We must now compute an estimate of the right hand side of equation ( 8 ). Note that $y_{t+i}$ also follows: ...

$$
\begin{aligned}
& y_{t+i}=a_{1} y_{t+i-1}+a_{2} y_{t+i-2} \quad \text { for } i>0 \\
& y_{t}=\xi_{t} ; y_{t+i}=0 \quad \text { for } i<0
\end{aligned}
$$


Multiplying equation ( 9 ) by $\theta^{i+1}$, and summing from one to infinity yields:

$$
\begin{aligned}
& \sum_{i=1}^{\infty} \theta^{i+1} y_{t+i}=a_{1} \theta\left(\sum_{i=1}^{\infty} \theta^{i+1} y_{t+i}+\theta \xi_{t}\right)+a_{2} \theta^{2}\left(\sum_{i=1}^{\infty} \theta^{i+1} y_{t+i}+\theta \xi_{t}\right) \\
\Rightarrow \quad & \sum_{i=1}^{\infty} \theta^{i+1} y_{t+i}=\left(1-\theta a_{1}-\theta^{2} a_{2}\right)^{-1}\left(\theta \xi_{t}\left(\theta a_{1}+\theta^{2} a_{2}\right)\right) \\
\Rightarrow \quad & \sum_{i=0}^{\infty} \theta^{i+1} y_{t+i}=\left(1-\theta a_{1}-\theta^{2} a_{2}\right)^{-1} \theta \xi_{t}
\end{aligned}
$$

so that

$$
\mathrm{v}_{c}^{\max }=\sigma_{\xi}^{2}\left(1-\theta \mathrm{a}_{1}-\theta^{2} \mathrm{a}_{2}\right)^{-2} \theta^{2}
$$

We have estimates of $a_{1}, a_{2}$ and $\sigma_{\xi}^{2}$. We need only a value for $\theta$, or recalling that $\theta=(1+r)^{-1}$ a value for the interest rate: Following Shiller, we assume $r=5 \%$, so that $\theta=.95$. This gives an estimated upper bound: $\hat{v}_{c}^{\max }=9.08$. We now need to compute the sample conditional variance, given by $E(p(R-r))^{2}$. This also has been computed by shiller. It is: $\hat{\mathrm{V}}_{\mathrm{c}}=653.83$.

The point estimates again violate the bound. Is the violation significant? We need to compute the variances of the above estimates. Conditional on the value of $\theta$, and assuming that $\xi_{t}$ is normally distributed, it is straightforward to show that asymptotically: $\operatorname{Var}\left(\hat{\mathrm{V}}_{\mathrm{C}}^{\max }\right)=15.13$ and $\operatorname{Var}\left(\hat{\mathrm{V}}_{\mathrm{c}}\right)=7843.92$. The asumptotic variance of $\left(\hat{\mathrm{V}}_{\mathrm{c}}^{\max }-\hat{\mathrm{V}}_{\mathrm{c}}\right)$ is thus: $15.13+7843.92-2 \operatorname{cov}\left(\hat{\mathrm{V}}_{\mathrm{c}}^{\max }, \hat{\mathrm{V}}_{\mathrm{c}}\right)$. We won't calculate the covariance term, but by assuming that $\hat{V}_{c}^{\max }$ and $\hat{V}_{c}$ are perfectly negatively correlated, we obtain a lower bound on the covariance term of -344.50. This implies an upper bound on the standard error of $\left(\hat{v}_{c}^{\max }-\hat{v}_{c}\right)$ of 92.46. Thus the t-statistic on $\left(\hat{v}_{c}^{\max }-\hat{v}_{c}\right)$ is greater, in absolute value, than 6.97, indicating that the data violate the bound given in (8) at any reasonable significance level. 


\section{Cross-covariances of $p$ and $x$}

The intuition that bubbles decrease the relation between $p$ and $x$ can be made rigorous as follows. Assume that there are no bubbles, so that:

$$
\begin{aligned}
p_{t} & =\sum_{i=0}^{\infty} \theta^{i+1} E\left(x_{t+i} \mid \Omega_{t}\right) \\
& =\sum_{i=0}^{\infty} \theta^{i+1} x_{t+i}+u_{t} ; E\left(u_{t} \mid \Omega_{t}\right)=0
\end{aligned}
$$

Then the unconditional variance of $p$ is given by:

$$
\begin{aligned}
v_{u} & =E\left[\left(\sum_{i=0}^{\infty} \theta^{i+1} x_{t+i}+u_{t}\right)\left(p_{t}\right)\right] \\
& =\sum_{i=0}^{\infty} \theta^{i+1} \operatorname{cov}\left(p_{t} x_{t+i}\right)+E\left(u_{t} p_{t}\right)
\end{aligned}
$$

By construction of $u_{t}, E\left(u_{t} p_{t}\right)=0$, so that:

$$
\sum_{i=0}^{\infty} \theta^{i+1} \operatorname{cov}\left(p_{t} x_{t+i}\right) / v_{u}=\frac{\sigma_{x}}{\sigma_{p}} \sum_{i=0}^{\infty} \theta^{i+1} \rho\left(p_{t} x_{t+i}\right)=1
$$

Under the null hypothesis, the relation of $p$ and $x$ is such that the appropriately weighted sum of correlations between $p$ and $x$, multiplied by the ratio of the standard deviation of $x$ to the standard deviation of $p$, is equal to unity. It is likely to be smaller if there are bubbles. Using the same sample, we can estimate this ratio. The two components are:

$$
\begin{aligned}
& \left(\sum_{i=0}^{\infty} \theta^{i+1} \hat{\operatorname{cov}}\left(p_{t} x_{t+i}\right)\right)=176.10 \text {, while from above: } \\
& \hat{v}_{u}(p)=2512 .
\end{aligned}
$$

Again, point estimates strongly suggest rejection of the null hypothesis. We have not carried out a formal test; this could be calculated using spectral techniques as in singleton [1981]. The result is suggestive of bubbles, with the same caveats as above. 
Section V Looking for Bubbles, II

We discuss in this section the use of two other types of tests for bubbles: runs tests and tail tests. Both refer to the distribution of innovations in prices, $p-E\left(p \mid \Omega_{-1}\right)$, or equivalently the distribution of excess returns $p(R-r)$. A run is a sequence of realizations of a random variable with the same sign.

The bubble component, $\varepsilon_{t}$, of the price innovation appears likely to have both runs and a distribution with fat tails. If bubbles grow for a while and then crash, the innovations in the bubble will tend to be of the same sign while the bubble continues, then reverse sign when a crash occurs. The runs for the bubble innovation will then tend to be longer than for a purely random sequence, making the total number of runs over the sample smaller. Crashes will produce large outliers so that the distribution of innovations will have fat tails. (i.e. the distribution will be leptokurtic).

Those are, however, characteristics of the bubble innovations, which are not observable. Price innovations, which are observable are the sum of bubble innovations, $\varepsilon$, and market fundamental innovations, $n$. To attribute characteristics of price innovations to bubbles implies imposing restrictions on the distribution of $n$. We now consider whether these may be reasonable.

\section{Runs Tests}

Runs in innovations can only arise from a skewed distribution. If we assume that $n$ has a symmetric distribution, we can then attribute runs in price innovations to bubbles. Is it reasonable to assume that $n$, the market fundamental innovation has a symmetric distribution? It may not be, even if $x$ has a symmetric distribution, as the following example shows; suppose that:

$$
x_{t}=\xi_{1 t}+\xi_{2 t-1}, \xi_{1}, \xi_{2} \text { independent and white, }
$$

with $\quad\left(\xi_{1 t}, \xi_{2 t}\right) \varepsilon \Omega_{t}, \xi_{1 t} \notin \Omega_{t-1}$ 
Suppose further that the probability derisity of $\xi_{1 t}, f\left(\xi_{1}\right)$, is skewed to the right and the density of $\xi_{2 t}$ is given by $g\left(\xi_{2}\right)=f\left(-\xi_{1}\right)$. In this case $x$ has a symetric distribution, but even in the absence of bubbles $p_{t}=\theta\left(\xi_{1 t}+\xi_{2 t-1}+\theta \xi_{2 t}\right)$ is skewed to the left and $p_{t}-E\left(p_{t} \mid \Omega_{t-1}\right)=\theta\left(\xi_{1 t}+\theta \xi_{2 t}\right)$ is skewed to the right. Even if $\eta$ is symmetric, runs tests may have only minimal power against bubbles. This is because bubbles do not necessarily generate long runs. Consider the second example of a bubble given in Section II. If $\pi$, the probability of the bubble continuing is unity, then the bubble innovation is simpiy $\mu_{t}$ which could have a symmetric distribution. Even if $\pi$ is different from one and the distribution of $\mu$ is symmetric, we may still find no fewer runs than for a series of independent random variables drawn from a symmetric distribution. If $\pi \neq 0$, the bubble innovation is, in this case

$$
\begin{aligned}
\varepsilon_{t} & =\mu_{t}+c_{t-1}(1-\pi)(\theta \pi)^{-1} & \text { with probability } \pi \\
& =\mu_{t}-c_{t-1} \theta^{-1} & \text { with probability } 1-\pi
\end{aligned}
$$

so that:

$$
\begin{aligned}
& \operatorname{Prob}\left(\varepsilon_{t}>0 \mid \varepsilon_{t-1}>0\right)=\pi \operatorname{Prob}\left(\mu_{t}>-c_{t-1}(1-\pi)(\theta \pi)^{-1} \mid \varepsilon_{t-1}>0\right) \\
& +(1-\pi) \operatorname{Prob}\left(\mu_{t}>+c_{t-1} \theta^{-1} \mid \varepsilon_{t-1}>0\right)
\end{aligned}
$$

Note that for $\pi=1 / 2$, this is just

$$
\begin{gathered}
\operatorname{Prob}\left(\varepsilon_{t}>0 \mid \varepsilon_{t-1}>0\right)=1 / 2 \operatorname{Prob}\left(\mu_{t}>-c_{t-1} \theta^{-1} \mid \varepsilon_{t-1}>0\right) \\
+1 / 2 \operatorname{Prob}\left(\mu_{t}>+c_{t-1} \theta^{-1} \mid \varepsilon_{t-1}>0\right)=1 / 2
\end{gathered}
$$

Thus, for $\pi=1 / 2$ or $\pi=1$, a runs test has minimal power against this type of bubble, and it has very low power for values of $\pi$ close to $1 / 2$ or 1 . 
Not detered by these caveats, we nevertheless calculated the distribution of runs for weekly innovations in the price of gold. We assumed, plausibly, that holding gold during the sample period, 1975 - 1981 was not providing any direct dividend, so that $x_{t}$ was equal to zero. In this case, the innovations are given by:

$$
p-E\left(p \mid \Omega_{-1}\right)=p(R-r)=p-(1+r) p_{-1}
$$

We used weekly gold prices (Englehart) for the period January, 1975, to June, 1981. In calculating the innovations we relaxed the assumption of a constant interest rate. The rate of interest that we used was the one week rate of return on Treasury bills which matured at time $t$.

The results of the runs tests are presented in Table 1 . They reveal no evidence indicating the presence of bubbies. This is somewhat surprising given the increase in prices at the end of 1979. In Figure 1 we have plotted the excess returns from August 1977 until the end of the sample. The figure clearly shows that the increase in late 1979 and early 1980 was not a steady one. Between the beginning of September 1980 and the middle of January 1981, gold prices rose from $\$ 335$ per ounce to $\$ 751$ per ounce. During this 19 week period, 13 of the week-to-week excess returns were positive, while 6 of the excess returns were negative.

\section{Tail Tests}

The bubble considered above (with $\pi \neq 1$ or $\pi \neq 0$ ) will at times explode or crash. While the bubble is growing it will generate small positive excess returns, which will be followed at the time of the crash by a large negative excess return. The distribution of innovations for this type of bubble will therefore be leptokurtic. This suggests that a large coefficient of kurtosis for price innovations might indicate the presence of bubbles.

Thus, if we assume that market fundamental innovations are not leptokurtic, 
TABLE 1

Runs for Gold Excess Returns

334 Observations

\begin{tabular}{|c|c|c|c|c|}
\hline Length & + & - & Total & for Random Sequence \\
\hline 1 & 45 & 44 & 99 & 83.50 \\
\hline 2 & 18 & 23 & 51 & 41.75 \\
\hline 3 & 5 & 4 & 9 & 20.88 \\
\hline 4 & 5 & 4 & 9 & 10.44 \\
\hline 5 & 2 & 4 & 6 & 5.22 \\
\hline 6 & 3 & 0 & 3 & 2.61 \\
\hline 7 & 1 & 0 & 1 & 1.30 \\
\hline 8 & 1 & 1 & 2 & .65 \\
\hline 9 & 1 & 0 & 1 & .33 \\
\hline 10 & 1 & 1 & 2 & .16 \\
\hline 11 & 0 & 0 & 0 & .08 \\
\hline 12 & 0 & 0 & 0 & .04 \\
\hline Total & 82 & 81 & 163 & 168 \\
\hline
\end{tabular}




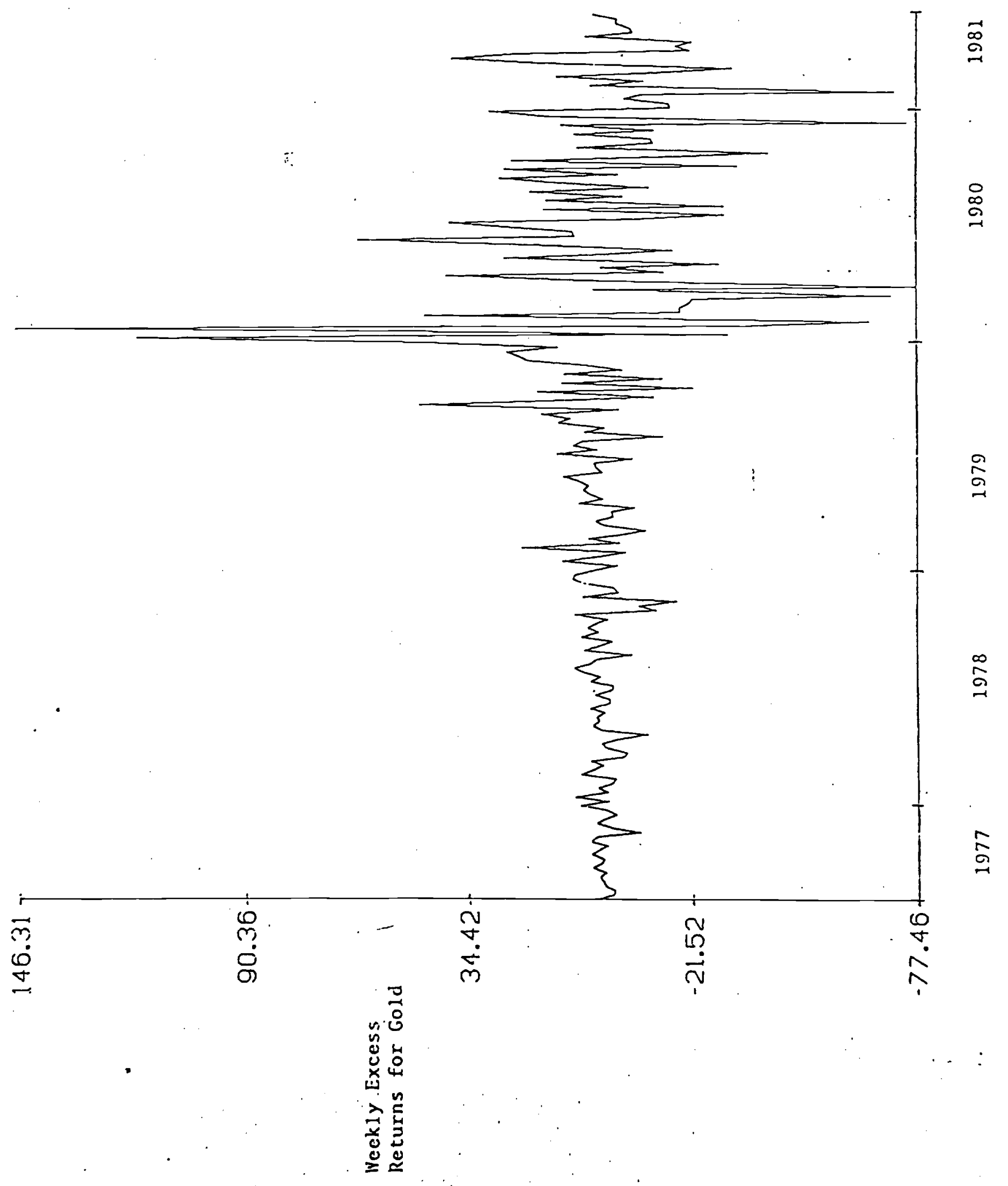


we can attribute fat tails in excess returns to the presence of bubbles. The problem is, however, the same as for runs. Even if the innovations in $x$ are not leptokurtic, the market fundamental innovations may well be. As Shiller [1981] points out, this will be the case in particular if information about future $x$ 's comes in lumps.

Again, despite the caveats, we computed the coefficient of kurtosis for excess returns for our weekly gold series. Because the series was heteroskedastic (see Figure 1) we computed the coefficient using data from the beginning of the sample to October, 1979, and from October, 1979, until the end of the sample. The kurtosis coefficients were 7.19 and 6.67 respectively. These are much higher than the normal distribution, which has a coefficient of kurtosis equal to 3 . It is also much higher than the coefficient for 25 week T-bills, whose values over the same periods were 4.30 and 3.36 . Whether this is due to fat tailed fundamentals, to a particular information structure, or to the presence of bubbles is impossible to tell.

The limits of these two types of tests have been emphasized. The results are nevertheless intriguing. The lack of runs suggests that if there were bubbles, they were either very long lived ( $\pi$ close to unity) or short lived $(\pi$ close to $1 / 2$, so that the average duration is two weeks). The very high coefficient of kurtosis on the other hand suggests either very leptokurtic market fundamentals or the existence of bubbles. 


\section{Section VI Conclusions}

Speculative bubbles are not ruled out by rational behavior in financial markets and are likely to have real effects on the economy.

Testing for speculative bubbles is not easy. Rational bubbles can follow many types of processes. We have shown that certain bubbles will cause violation of variance bounds implied by a class of rational expectations models. Empirical evidence is presented which demonstrates that these bounds are violated. We also noted that other alternatives (e.g. irrationality) would cause violation of these bounds, and our results must be viewed in light of this.

Other tests for bubbles were suggested when only price data is available. Our discussion demonstrated that these tests may have low power. 


\section{Footnotes}

(1) This indeterminacy arises not only in arbitrage conditions, but in all models in which expectations of future variables affect current decisions. It is the subejct of much discussion currently in macroeconomics, under the label of "non uniqueness."

(2) In some models, such as the model used by Cagan [1956], a condition similar. to (1) holds with $\mathrm{p}_{t}$ being the logarithm of the price. As a logarithm can be negative, the argument used in this paragraph does not apply.

(3) The first example of a bubble type phenomenon in a general equilibrium model was given by Hahn [1966]. In his model, however, bubbles imply that a price becomes negative in finite time. As this is impossible, rational expectations and general equilibrium implications exclude the presence of bubbles in his model.

(4) The assumptions of risk neutrality and of a constant interest rate, inessential in previous sections, are essential in this one. The moment tests are only valid if they hold. 


\section{References}

Cagan, Phillip, "The Monetary Dynamics of Hyperinflation." In Studies in the Quantity Theory of Honey, edited by Milton Friedman, Chicago: University of Chicago Press, 1956.

Flood, Robert P. and Peter M. Garber, "Market.Fundamentals versus Price-level Bubbles: The First Tests." Journal of Political Economy 88, no. 4 (August, 1980): pp. 745-770.

Hahn, F. H. "Equilibrium Dynamics with Heterogenous Capital Goods," Quarterly Journal of Economics, 80 (November, 1966): pp. 633-646.

Kindleberger, C. P. Manias, Panics, and Crashes, Basic Books, N.Y., 1978.

Poterba, J. M. "Inflation, Income Taxes, and Owner Occupied Housing." Thesis, Harvard University, March, 1980.

Shiller, R. J. "Do Stock Prices Move too Much to be Justified by Subsequent Changes in Dividends?" American Economic Review, 71, no. 3 (June, 1981).

Singleton, K. J. "Expectations Models of the Term Structure and Implied Variance Bounds." Journal of Political Economy, 88, no. 6 (December, 1980).

Thomas, G. and M. Morgan Witts, The Day the Bubble Burst, Doubleday, N.Y., 1979. 